\title{
CONVERGENCE OF A SPECIAL SET OF TRIANGLES
}

Dedicated to Sherman Kopald Stein (11 August 1926-...) for his pleasant sense of humor and mathematical wisdom that the third author enjoyed as a colleague during the late 1980s at the University of California, Davis

\section{MOSHE STUPEL ${ }^{1}$, AVI SIGLER ${ }^{2}$ and JAY JAHANGIRI ${ }^{3}$}

${ }^{1}$ Shannan Academic College of Education

Gordon Academic College of Education

Haifa

Israel

e-mail: stupel@bezeqint.net

2Shannan Academic College of Education

Haifa

Israel

e-mail: avibsigler@gmail.com

${ }^{3}$ Mathematical Sciences

Kent State University

Kent, Ohio

USA

e-mail: jjahangi@kent.edu

\begin{abstract}
An explicit expression for the convergence point of a given set of triangles is introduced leading to an open interval locus of convergence that contains the centroid of the triangles.
\end{abstract}

2010 Mathematics Subject Classification: Primary: 97G40; Secondary: 97G50.

Keywords and phrases: geometric convergence, barycentric coordinates, Ceva's theorem, Pappus configuration.

Received March 8, 2018; Revised April 16, 2018

(C) 2018 Scientific Advances Publishers 


\section{Introduction}

Using coordinate systems such as rectangular, polar, spherical and cylindrical require an origin but at times we need to locate points relative to existing points or local coordinates rather than the origin [1-4].

Mobius assigned coordinates (barycentric coordinates) to the point at which the center of gravity, or barycentre, was located, reflecting the ratio of weights attached to the ends of a given rod. He then extended this idea to a system of three weighted points, forming a triangle and pointing out that the center of gravity will remain the same if the weights are increased or decreased by a common factor $[5,6]$.

Central to barycentric coordinates are the concept of concurrency of lines in a triangle and Ceva's theorem that "Given any triangle, the segments from each vertex to the opposite sides of the triangle are concurrent precisely when the product of the ratios of the pairs of segments formed on each side of the triangle is equal to 1 " [5, 7].

Ceva's theorem is used in the proofs of many well-known theorems including the theorem of Napolean's point, the theorem of Fermat's point, and the theorem of the Nagel point [8]. Barycentric coordinates are a fundamental tool for a wide variety of applications employing triangular meshes, underlining the definition of higher-order basis functions, the Bézier triangle in computer aided-design, and many interpolation and shading techniques in computer graphics $[9,10]$.

In the present paper, we use the convergence of a special set of triangles leading to the discovery of an explicit expression for the convergence point and the understanding that this point can be constructed using a compass and a straightedge ruler, even if the convergence process is infinite. These results are then generalized to Pappus triangles and generalized Pappus triangles [11-13]. Finally, we obtain an interesting open interval locus of convergence that contains the centroid of the triangle. 


\section{Terminology and Notation}

The interior point $D$ of the triangle $\triangle A B C$ (Figure 1)

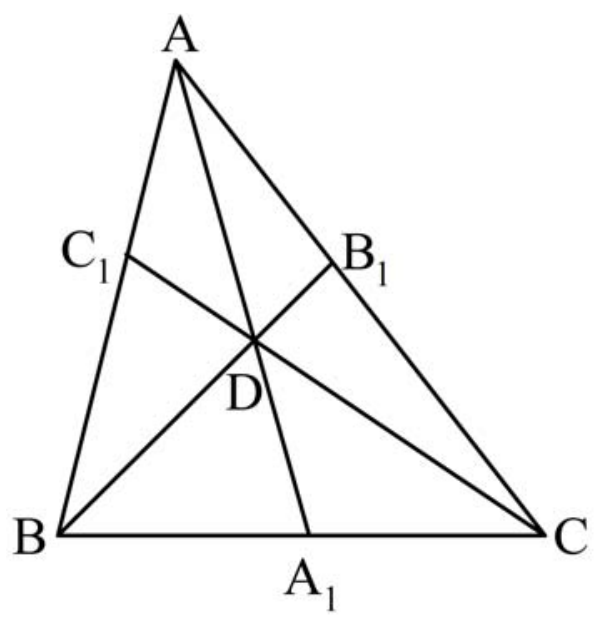

Figure 1. Representation of the point $D$.

shall be represented by two methods.

(a) Using the barycentric coordinates $\left(\lambda_{1}, \lambda_{2}, \lambda_{3}\right)$, we obtain

$$
\begin{aligned}
& x_{D}=\lambda_{1} x_{A}+\lambda_{2} x_{B}+\lambda_{3} x_{C}, \\
& y_{D}=\lambda_{1} y_{A}+\lambda_{2} y_{B}+\lambda_{3} y_{C}, \text { where } \\
& \lambda_{1}+\lambda_{2}+\lambda_{3}=1, \text { and } \\
& \lambda_{1} \geq 0, \lambda_{2} \geq 0, \lambda_{3} \geq 0 .
\end{aligned}
$$

(b) Using the Ceva coordinates $(\alpha, \beta, \gamma)$, we obtain

$A A_{1}, B B_{1}$, and $C C_{1}$ which are three cevians that pass through $D$ so that $\frac{B A_{1}}{A_{1} C}=\alpha, \frac{C B_{1}}{B_{1} A}=\beta$, and $\frac{A C_{1}}{C_{1} B}=\gamma$, where

$$
\alpha \beta \gamma=1, \quad \alpha \geq 0, \quad \beta \geq 0, \quad \gamma \geq 0 .
$$


Known are the relations between the barycentric coordinates and the Ceva coordinates

$$
\left.\begin{array}{c}
\lambda_{1}=\frac{\alpha \beta}{\alpha \beta+\alpha+1} \\
\lambda_{2}=\frac{1}{\alpha \beta+\alpha+1} \\
\lambda_{3}=\frac{\alpha}{\alpha \beta+\alpha+1}
\end{array}\right\},
$$

\section{The Convergence Process}

The convergence process used here are presented in two phases.

\section{Case I: The Pappus configuration}

Select the points $C_{1}, B_{1}, A_{1}$, respectively, on the sides $C A, B C$, and $A B$ so that $\frac{A A_{1}}{A_{1} B}=\frac{B B_{1}}{B_{1} C}=\frac{C C_{1}}{C_{1} A} \equiv \alpha$. Similarly, select the points $C_{2}, B_{2}, A_{2}$, respectively, on the sides $C_{1} A_{1}, B_{1} C_{1}$ and $A_{1} B_{1}$ so that $\frac{A_{1} A_{2}}{A_{2} B_{1}}=\frac{B_{1} B_{2}}{B_{2} C_{1}}=\frac{C_{1} C_{2}}{C_{2} A_{1}} \equiv \alpha$.

Repeating this process $(N+1)$-times ( $N$ is a whole number), we arrive at the points $C_{N+1}, B_{N+1}, A_{N+1}$, which are, respectively, on the sides $C_{N} A_{N}, B_{N} C_{N}$, and $A_{N} B_{N}$, so that they divide the sides of the triangle $A_{N} B_{N} C_{N}$ at a ratio that equals $\alpha$.

Note that the triangle $A_{0} B_{0} C_{0}$ is the original triangle $A B C$ and the sides $A_{0} B_{0}, B_{0} C_{0}$, and $C_{0} A_{0}$ are the sides of the original triangle $A B C$. 
The famous Pappus' theorem claims that the triangles $\triangle A B C$ and $\triangle A_{1} B_{1} C_{1}$ have a common centroid [14]. Therefore, it is clear that the triangles $\triangle A_{1} B_{1} C_{1}$ and $\triangle A_{2} B_{2} C_{2}$ also have a common centroid, and so on and so forth, up to the triangles $A_{N} B_{N} C_{N}$ and $A_{N+1} B_{N+1} C_{N+1}$. A continuous repetition of this process leads to the convergence of the triangles $A_{N+1} B_{N+1} C_{N+1}$ to a single centroid point as $N$ approaches infinity and therefore we have the following:

Claim 1. $\cap \triangle A_{N+1} B_{N+1} C_{N+1}$ is the centroid point of $\triangle A B C$, as $N$ tends to infinity.

\section{Case II: Generalization}

The points $C_{1}, B_{1}, A_{1}$ lie, respectively, on the sides $C A, B C$, and $A B$ of the triangle $\triangle A B C$ so that

$$
\frac{B B_{1}}{B_{1} C} \equiv \alpha, \quad \frac{C C_{1}}{C_{1} A} \equiv \beta, \quad \frac{A A_{1}}{A_{1} B} \equiv \gamma(\text { Figure } 2) .
$$

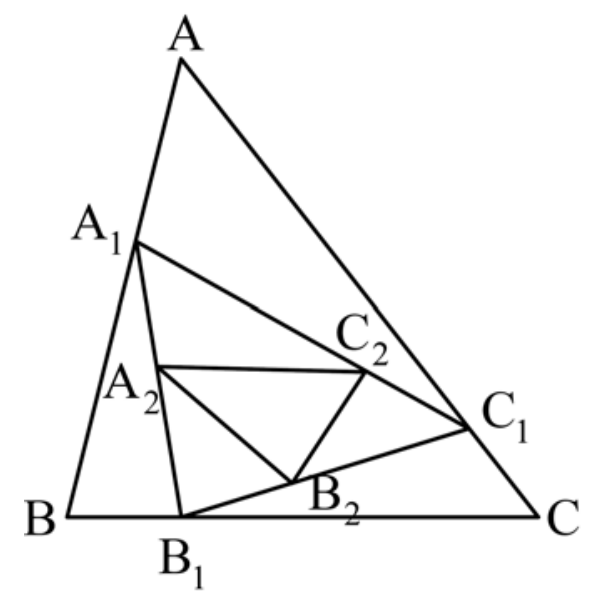

Figure 2. Convergence of Pappus triangles. 
The points $C_{2}, B_{2}, A_{2}$ lie, respectively, on the sides $C_{1} A_{1}, B_{1} C_{1}$, and $A_{1} B_{1}$ of the triangle $\Delta A_{1} B_{1} C_{1}$ so that

$$
\frac{B_{1} B_{2}}{B_{2} C_{1}} \equiv \alpha, \quad \frac{C_{1} C_{2}}{C_{2} A_{1}} \equiv \beta, \quad \frac{A_{1} A_{2}}{A_{2} B_{1}} \equiv \gamma
$$

Repeating this process $(N+1)$-times, we conclude that the points $C_{N+1}$, $B_{N+1}, A_{N+1}$, respectively, lie on the sides $C_{N} A_{N}, B_{N} C_{N}, A_{N} B_{N}$ so that

$$
\frac{B_{N} B_{N+1}}{B_{N+1} C_{N}} \equiv \alpha, \quad \frac{C_{N} C_{N+1}}{C_{N+1} A_{N}} \equiv \beta, \quad \frac{A_{N} A_{N+1}}{A_{N+1} B_{N}} \equiv \gamma
$$

Next we state and prove.

Claim 2. $\cap \triangle A_{N+1} B_{N+1} C_{N+1}=S$; a point of $\triangle A B C$, with the barycentric coordinates

$$
\begin{aligned}
\left(\lambda_{1}^{S}, \lambda_{2}^{S}, \lambda_{3}^{S}\right)=\left(\frac{\alpha \beta(\gamma+1)}{\alpha \beta(\gamma+1)+\beta \gamma(\alpha+1)+\alpha \gamma(\beta+1)},\right. & \\
& \left.\frac{\beta \gamma(\alpha+1)}{\alpha \beta(\gamma+1)+\beta \gamma(\alpha+1)+\alpha \gamma(\beta+1)}, \frac{\alpha \gamma(\beta+1)}{\alpha \beta(\gamma+1)+\beta \gamma(\alpha+1)+\alpha \gamma(\beta+1)}\right),
\end{aligned}
$$

or with the Ceva coordinates

$$
\left(\lambda_{1}^{S}, \lambda_{2}^{S}, \lambda_{3}^{S}\right)=\left(\frac{\alpha(\beta+1)}{\beta(\alpha+1)}, \frac{\beta(\gamma+1)}{\gamma(\beta+1)}, \frac{\gamma(\alpha+1)}{\alpha(\gamma+1)}\right)
$$

Proof. We transform the triangle $A B C$ by an affine transformation to a triangle in which $A, B$, and $C$ have the coordinates $A(0,1), B(0,0)$, and $C(1,0)$.

Next, we transform the points $A, C, B$ by an affine transformation to respective points $C_{1}, B_{1}, A_{1}$ so that $\frac{B B_{1}}{B_{1} C}=\alpha, \frac{C C_{1}}{C_{1} A}=\beta$, and $\frac{A A_{1}}{A_{1} B}=\gamma$.

Repeating this process $(N+1)$-times, we note that these affine transformations preserve the simple ratio where the triangle $\Delta A_{N} B_{N} C_{N}$ 
shall be transformed into the triangle $\Delta A_{N+1} B_{N+1} C_{N+1}$ so that the division ratios of the corresponding sides are $\alpha, \beta$, and $\gamma$, as required.

The affine transformation $f$ that transforms $A, C, B$ to $C_{1}, B_{1}, A_{1}$, is denoted by $f(\triangle A B C) \equiv \triangle A_{1} B_{1} C_{1}$ (Figure 3 ). This is a continuous function from the convex domain $\triangle A B C$ to the convex domain $\triangle A_{1} B_{1} C_{1}$ that is contained in $\triangle A B C$. Therefore $f$ has a fixed point $S$. This point $S$ shall be the only common point of the triangles $\bigcap \Delta A_{N+1} B_{N+1} C_{N+1}$.

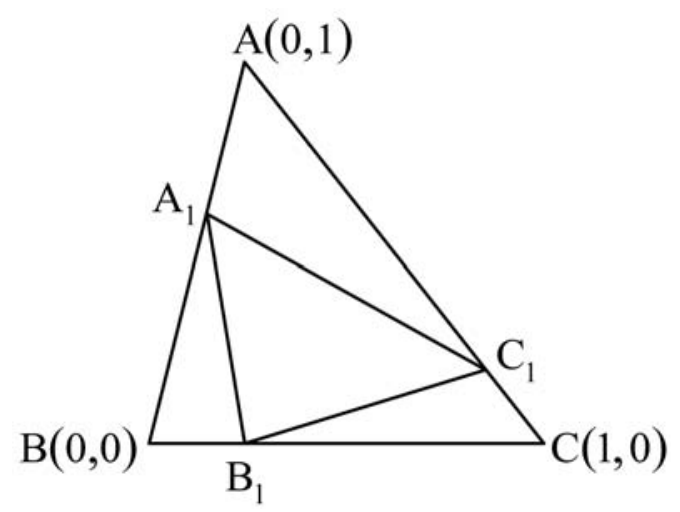

Figure 3. Affine transformation $f(\triangle A B C)=\triangle A_{1} B_{1} C_{1}$.

To derive the coordinates of the point $S$, observe that the coordinates of $A_{1}, B_{1}$, and $C_{1}$ are

$$
A_{1}=\left(0, \frac{1}{\gamma+1}\right), B_{1}=\left(\frac{\alpha}{\alpha+1}, 0\right), C_{1}=\left(\frac{1}{\beta+1}, \frac{\beta}{\beta+1}\right) .
$$

The fixed point for $f$ has the same barycentric coordinates in $\triangle A B C$ and $\triangle A_{1} B_{1} C_{1}$. Therefore it is a solution of the following system of equation:

$$
x_{S}=\underbrace{\lambda_{3} \cdot 1+\lambda_{1} \cdot 0+\lambda_{2} \cdot 0}_{\Delta A B C}=\underbrace{\lambda_{1} \cdot 0+\lambda_{2} \cdot \frac{\alpha}{\alpha+1}+\lambda_{3} \cdot \frac{1}{\beta+1}}_{\Delta A_{1} B_{1} C_{1}} .
$$


In the other words,

$$
\begin{gathered}
\lambda_{3}=\lambda_{2} \cdot \frac{\alpha}{\alpha+1}+\frac{\lambda_{3}}{\beta+1}, \\
\lambda_{1}=\frac{\lambda_{1}}{\gamma+1}+\frac{\lambda_{3} \beta}{\beta+1} .
\end{gathered}
$$

From (5), it follows that

$$
\alpha_{S}=\frac{\lambda_{3}}{\lambda_{2}}=\frac{\alpha(\beta+1)}{\beta(\alpha+1)}
$$

From (6), it follows that

$$
\beta_{S}=\frac{\lambda_{1}}{\lambda_{3}}=\frac{\beta(\gamma+1)}{\gamma(\beta+1)} .
$$

And clearly, we obtain

$$
\gamma_{S}=\frac{\lambda_{2}}{\lambda_{1}}=\frac{\gamma(\alpha+1)}{\alpha(\gamma+1)}
$$

A simple calculation, using Equation (1) gives $\lambda_{1}^{S}=\frac{\alpha \beta(\gamma+1)}{\alpha \beta(\gamma+1)+\beta \gamma(\alpha+1)+\alpha \gamma(\beta+1)}$. The process for $\lambda_{2}^{S}$ and $\lambda_{3}^{S}$ are similar and will be omitted.

Note. For given $\alpha, \beta$, and $\gamma$, the point $S$ can be constructed using a compass and a straightedge ruler (though the convergence process is infinite). An accurate description of segment construction, by using compass and straightedge, based on expressions whose representation is by segments, can be found in references $[1,2]$. 
Next we deal with three particular cases (Figure 4).

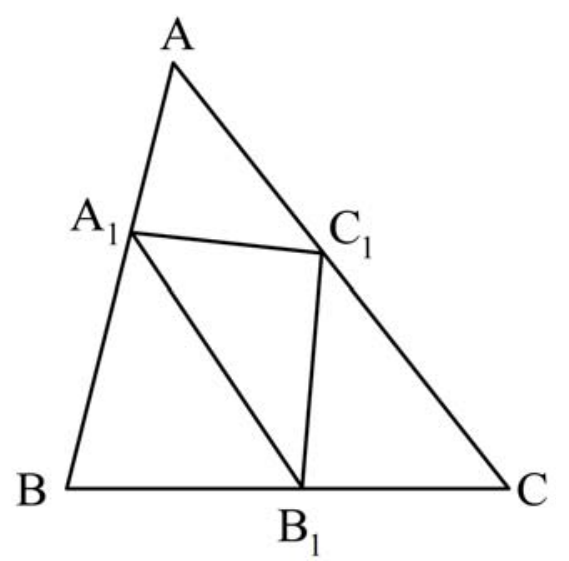

Figure 4. The case of the middle of the segments.

\section{First particular case}

If $\alpha=\beta=\gamma=1$, then the convergence is at the centroid of $\triangle A B C$.

\section{Second particular case (Pappus)}

If $\alpha=\beta=\gamma=k$, where $k(0<k<\infty)$ is fixed, then $S$ is the centroid of $\triangle A B C$ because $\alpha_{S}=\beta_{S}=\gamma_{S}=1$.

\section{Third particular case (Generalized Pappus)}

If $\frac{B B_{1}}{B_{1} C}=k \alpha, \frac{C C_{1}}{C_{1} A}=k \beta$ and $\frac{A A_{1}}{A_{1} B}=k \gamma$, where $k(0<k<\infty)$ is fixed, then

$$
\left.\begin{array}{l}
\alpha_{S}=\frac{\alpha(k \beta+1)}{\beta(k \alpha+1)} \\
\beta_{S}=\frac{\beta(k \gamma+1)}{\gamma(k \beta+1)} \\
\gamma_{S}=\frac{\gamma(k \alpha+1)}{\alpha(k \gamma+1)}
\end{array}\right\} .
$$

In the other words, aside from the Pappus' case, the point $S$ depends on $k$ and is therefore denoted by $S_{(k)}$. 
Finally, we state and prove our

Claim 3. The locus of $S_{(k)}$ where $k(0<k<\infty)$ is fixed, is the open interval straight-line segment bounded by two points $M_{1}$ and $M_{2}$ where $M_{1}$ is the centroid of the triangle and $M_{2}$ has the barycentric coordinates

$$
\lambda_{1}{ }^{M_{2}}=\frac{\alpha \beta}{\alpha \beta+\beta \gamma+\gamma \alpha}, \lambda_{2}{ }^{M_{2}}=\frac{\beta \gamma}{\alpha \beta+\beta \gamma+\gamma \alpha}, \lambda_{3}{ }^{M_{2}}=\frac{\alpha \gamma}{\alpha \beta+\beta \gamma+\gamma \alpha} .
$$

Proof. When $k$ tends to zero in (7), we obtain the Ceva coordinates $M_{2}=\left(\frac{\alpha}{\beta}, \frac{\beta}{\gamma}, \frac{\gamma}{\alpha}\right)$, which means the barycentric coordinates

$$
\left(\lambda_{1}{ }^{M_{2}}, \lambda_{2}{ }^{M_{2}}, \lambda_{3}{ }^{M_{2}}\right)=\left(\frac{\alpha \beta}{\alpha \beta+\beta \gamma+\gamma \alpha}, \frac{\beta \gamma}{\alpha \beta+\beta \gamma+\gamma \alpha}, \frac{\alpha \gamma}{\alpha \beta+\beta \gamma+\gamma \alpha}\right) .
$$

When $k$ tends to infinity, we obtain $(1,1,1)=\left(\alpha_{M_{1}}, \beta_{M_{1}}, \gamma_{M_{1}}\right)$, or barycentric coordinates $\left(\lambda_{1} M_{2}, \lambda_{2}{ }^{M_{2}}, \lambda_{3} M_{2}\right)=\left(\frac{1}{3}, \frac{1}{3}, \frac{1}{3}\right)$. We now prove that $S_{(k)}$ is located on the segment connecting $M_{1}$ and $M_{2}$. It is enough to prove that

$$
\left|\begin{array}{ccc}
\lambda_{1}{ }^{M 1} & \lambda_{2}{ }^{M_{1}} & \lambda_{3}{ }^{M_{1}} \\
\lambda_{1}{ }^{M_{2}} & \lambda_{2}{ }^{M_{2}} & \lambda_{3}{ }^{M_{2}} \\
\lambda_{1}{ }^{S_{(k)}} & \lambda_{2}{ }^{S_{(k)}} & \lambda_{3}{ }^{S(k)}
\end{array}\right|=0,
$$

or

$$
\left|\begin{array}{ccc}
1 & 1 & 1 \\
\alpha \beta & \beta \gamma & \alpha \gamma \\
\alpha \beta(k \gamma+1) & \beta \gamma(k \alpha+1) & \alpha \gamma(k \beta+1)
\end{array}\right|=0,
$$


or

$\left|\begin{array}{ccc}1 & 1 & 1 \\ \alpha \beta & \beta \gamma & \alpha \gamma \\ k \alpha \beta \gamma & k \alpha \beta \gamma & k \alpha \beta \gamma\end{array}\right|=0$.

We will show that $S_{(k)}$ is located between $M_{1}$ and $M_{2}$. We connect $A$ to $M_{1}$ and $M_{2}$, and obtain the respective points of intersection $D_{1}$ and $D_{2}$, (Figure 5).

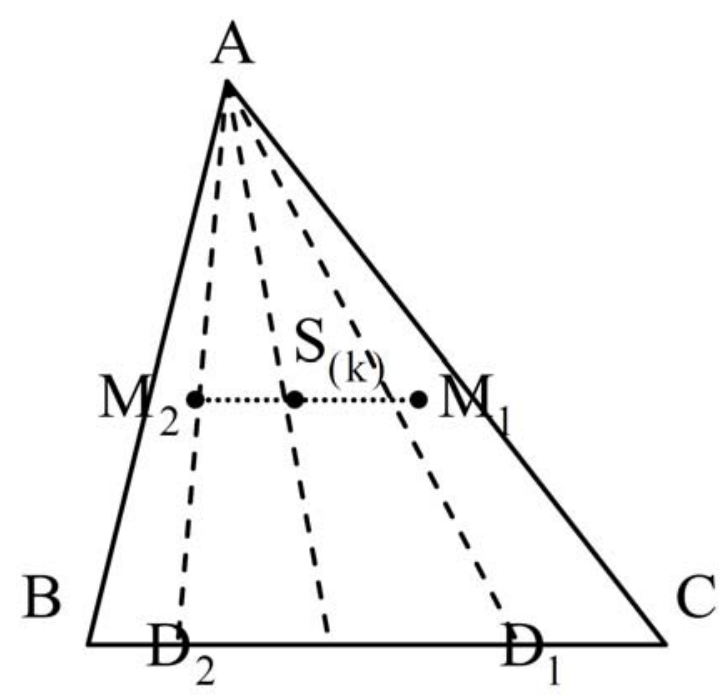

Figure 5. The location of the point $S_{(k)}$.

Without loss of generality, we assume that $M_{2}$ and $D_{2}$ are more to the left of $M_{1}$ and $D_{1}$, in the other words $\frac{\alpha}{\beta}<1$.

In this case $\frac{\alpha}{\beta}<\alpha_{S_{(k)}}=\frac{\alpha(\beta k+1)}{\beta(\alpha k+1)}<1$ and therefore $S_{(k)}$ is located between $M_{1}$ and $M_{2}$. 


\section{References}

[1] G. E. Martin, Geometric Constructions, Springer, New York (1998), 131-142.

[2] V. Oxman, M. Stupel and A. Sigler, Geometric constructions for geometric optics using a straightedge only, Journal for Geometry and Graphics 18(1) (2014), 73-79.

[3] M. Stupel, V. Oxman and A. Sigler, More on geometrical constructions of a tangent to a circle with a straightedge only, The Electronic Journal of Mathematics and Technology 8(1) (2014), 17-30.

[4] A. Sutton, Ruler and Compass: Practical Geometric Constructions, Walker \& Company, 2009.

[5] P. Lidberg, Barycentric and Wachspress Coordinates in two Dimensions: Theory and Implementation for Shape Transformations, Uppsala Universitet, U.U.D.M. Project Report, February 2011.

[6] S. K. Stein, Archimedes: What did he do Besides Cry Eureka, The Mathematical Association of America, Page 15, Washington, 1999.

[7] D. A. Brannan, M. F. Esplen and J. J. Gray, Geometry, Cambridge University Press, page 75, Cambridge, 2004.

[8] D. N. V. Krishna, The fundamental property of Nagel point: A new proof, Journal of Mathematical Sciences \& Mathematics Education 12(2) (2017), 21-28.

http://www.msme.us/2017-2-4.pdf

[9] R. M. Rustamov, Y. Lipman and T. Funkhouser, Interior distance using barycentric coordinates, Eurographics Symposium on Geometry Processing 28(5) (2009), 1279-1288.

DOI: https://doi.org/10.1111/j.1467-8659.2009.01505.x

[10] J. Vince, Mathematics for Computer Graphics, (4th Edition), Springer, London, Heidelberg, New York, Dordrecht, 2013. ISBN: 978-1-4471-6289-6.

[11] H. Eves, An Introduction to the History of Mathematics, Philadelphia: Saunders College Publishing, 1992.

[12] A. Kryftis, A Constructive Approach to Affine and Projective Planes, Ph.D. Thesis, University of Cambridge, 2015.

[13] B. D. S. McConnell, A Six-Point Ceva-Menelaus Theorem, 3 March 2014.

$$
\text { https://arxiv.org/pdf/1403.0478.pdf }
$$

[14] A. W. Goodman and G. Goodman, Generalizations of the theorems of Pappus, The American Mathematical Monthly 76(4) (1969), 355-366. 